\title{
Pilot Testing Of A Brief Pre-Consultation Screener For Improving The Identification And Discussion Of Medication Adherence In Routine Consultations
}

This article was published in the following Dove Press journal: Patient Preference and Adherence

\author{
John Weinman' \\ Imran Ali $\mathbb{D}^{\prime}$ \\ Anna Hodgkinson (1D ${ }^{2}$ \\ Martha Canfield ${ }^{3}$ \\ Christina Jackson' \\ 'Medicines Use Group, Institute of \\ Pharmaceutical Sciences, Kings College \\ London, London SEI 9NH, UK; ${ }^{2} \mathrm{NHS}$ \\ Lambeth Diabetes Team, Crown Dale \\ Medical Centre, London SEI 9 3NY, UK; \\ ${ }^{3}$ Health Psychology Section, Institute of \\ Psychiatry, Psychology and Neuroscience, \\ Kings College London, London SEI \\ 9RT, UK
}

\begin{abstract}
Objective: One of the challenges in being able to identify and manage medication adherence problems in routine practice is that patients are often reluctant to "admit to" nonadherence, particularly when asked in a direct way. The study reported in this paper has been designed as part of an attempt to address this problem by examining the value of a new brief medicines use screener in helping patients to identify and discuss adherence issues in a clinical setting.
\end{abstract}

Methods: 145 Patients with type 2 diabetes completed the new screener (MMWFU) together with an adherence self-report scale (MMAS4) and medication beliefs questionnaire (BMQ). Correlations between the scales were assessed together with an assessment of the sensitivity and specificity of the new screener.

Results: $126(88 \%)$ of the sample identified at least one medicines-related issue on the MMWFU, which showed strong correlations with the MMAS4 and BMQ Concerns scales, as well as good sensitivity and specificity against the MMAS4.

Conclusion: The results indicate that the new screener can serve as a fairly sensitive indicator of non-adherence and its determinants. Future studies will be needed to establish how well it performs in other clinical settings.

Keywords: medication adherence, brief screener, medication beliefs, adherence assessment

\section{Introduction}

Even though there is substantial evidence that many patients are not adherent to their medication, ${ }^{1}$ there are a number of challenges for clinicians in being able to detect and manage medication adherence problems in routine practice. Patients are often unwilling to "admit to" non-adherence, particularly when asked in a direct way. ${ }^{2}$ As a result, clinicians are often unaware of the extent and impact of the problem in their own patients. ${ }^{1}$

The study reported in this paper has been designed as part of an attempt to address the problem of adherence under-reporting and detection by examining the value of a brief medicines use screener in helping patients to surface possible adherence issues in a clinical setting and to provide the HCP with a straightforward way of identifying these. Although other brief adherence measures are available, ${ }^{3,4}$ these typically ask patients directly about their adherence. While this is helpful for indicating the presence of non-adherence, it is known that many patients can be somewhat intimidated by this and are reluctant to admit to not taking their medicines. $^{2}$ We therefore wanted to develop an easy-to-use scale, which did not
Correspondence: John Weinman Sciences, Kings College London, 5th Floor Franklin Wilkins Building, Stamford Street, London SEI 9NH, UK Tel +442078484839

Email john.weinman@kcl.ac.uk 
ask patients directly about their adherence but allowed them to indicate whether they were experiencing one or more of a range of issues, which could be indicative of non-adherence. We envisaged that such a measure could be used immediately prior to a consultation and would not only be acceptable to patients but would also allow the clinician to see whether their patients had any medicationrelated issues in order to discuss and hopefully resolve these.

Working with patients and clinicians, we have designed a brief screener, which enables patients to indicate whether they are experiencing any one or more of seven problems with their medicines. The seven problem areas were drawn from a recent paper which mapped out the main drivers of adherence/non-adherence using the Capability/Opportunity/ Motivation (COM-B) framework. ${ }^{5}$ Since the screening questionnaire does not directly ask about adherence, we hoped that it would help patients to identify a range of medicines-related issues, which could then be discussed with their HCP. Thus, the aims of this pilot study were to: (i) use the screener in a clinical setting to ascertain the range and extent of the problems which patients experience; (ii) assess the relation between ratings on the screener and scores on established measures of medication adherence and beliefs; (iii) assess the sensitivity and specificity of the screener against a standardized adherence self-report measure.

\section{Methods}

The study was designed as a service evaluation of medicines use for the local Diabetes Intermediate Care Team in South London. As such, we checked with the clinical lead of the team as to whether it needed to undergo a full ethics review but this was not deemed to be necessary as a service evaluation. Moreover, the patients were informed that the questionnaire was to help them think about how they were finding their diabetes medicines and that their answers would help the clinic to improve its service. Each patient was shown the questionnaire and was given time to choose whether to complete it or not.

\section{Patient Samples}

A total of 161 consecutive patients with Type 2 Diabetes, from four different community diabetes outpatient clinics in South London, were approached over a 6-week period to participate in the study. Of these, 12 refused to take part and 4 questionnaires completed were not returned. Thus,
145 patients self-completed the questionnaire, giving an overall response rate of 145/161 (90.1\%).

\section{Measures}

Patients' characteristics like information about age, gender and length of time since diagnosed with Type 2 diabetes were collected.

Making Medicines Work For You (MMWFU) is an 8item screener, which was developed to identify reasons why patients may have problems with their medicines. It was developed in consultation with patients and clinicians. The first 7 items were chosen to broadly map onto the range of adherence determinants classified by the COM-B framework for adherence. ${ }^{5}$ Permission to use the CMMWFU screener needs to be obtained from the corresponding author. Charges may apply depending on the sector and nature of the request. It has the following instructions: The questions below are to help you think about how you are finding the medicines for your diabetes. Your answers will help to understand what's important to people like you to improve our service. Please look at each item below and tick any that apply to you. Some examples of the first seven items are: "I sometimes forget to use the medicine(s)", "I have some worries about using the medicine(s)" and "I've found my own way to use the medicine (s) that suits me". Each item that was ticked was scored 1, giving a score range from 0-7 since only the first seven items were used to calculate the MMWFU score. The eighth item was an open (ie free text) item, which allowed patients to indicate any other issues.

Morisky Medication Adherence Scale (MMAS4) ${ }^{3}$ was used to assess adherence. It consists of four items, such as "When you feel better do you sometimes stop taking your diabetes medication(s)?" and "Do you ever forget to take your diabetes medication(s)?". Items are scored on a 2point scale $(0=\mathrm{No} ; 1=$ Yes $)$ to give a score range from 0 to 4 , and allowing patients to be categorized as adherent ( score $=0$ ) or non-adherent (score $=1-4)$, which we used as a benchmark for assessing the sensitivity and specificity of the MMWFU (see below).

Permission and training to use the MMAS4 were obtained by our department from Prof Donald E.Morisky, $\mathrm{ScD}$, ScM, MSPH, Department of Community Health Sciences, UCLA School of Public Health, 650 Charles E. Young Drive South, Los Angeles, CA 90095-1772.

Beliefs about Medicines Questionnaires-Specific $(\mathrm{BMQ})^{6}$ were used to assess patients' beliefs about the Necessity of their prescribed medication (5 items) and their 
Concerns about it (five items). All items have a 5-point Likert scale response option ( $1=$ strongly disagree to $5=$ strongly agree) giving a score range of 5-25 for each scale.

\section{Data Analysis}

Descriptive statistics were calculated using frequencies and percentages for categorical data, and means and standard deviations for continuous data. Polychoric correlation matrices were conducted to assess the association between MMWFU, MMAS4, BMQ Necessity and Concerns. Two cut-off points of the MMWFU were derived to detect adherence/non-adherence. The sensitivity and specificity were tested by calculating the proportion of participants who met the MMWFU cut-off scores and MMAS4 adherent/non-adherent score.

\section{Results}

The final sample consisted of 75 women (51.7\%) and 70 men $(48.3 \%)$ with a mean age of 59.8 (SD 11.8) years and a mean disease duration of 13.4 (SD 8.7) years. The mean scores (SDs) for the scales were: 1.86 (1.29) for the MMWFU, 18.68 (13.) for the BMQ-Necessity, 14.6 (SD 3.59) for BMQ-Concerns and 0.97 (1.03) for MMAS4. Using the MMAS4 adherence/non-adherence criterion, $57 \%$ patients were classified as non-adherent. The mean scores on the BMQ are broadly consistent with the data for a range of long-term conditions, including diabetes, described in the original paper, ${ }^{6}$ indicating that this group of patients had a fairly strong belief in the need for their treatment and relatively low concerns about it.

On the MMWFU screener, 17 patients (12\%) did not endorse any of the seven items, 51 (35\%) endorsed one item and $77(53 \%)$ endorsed 2 or more items. Table 1 shows the frequency of responses for MMWFU items. The most commonly endorsed items were: I sometimes forget to use the medicine and I have found my own way to use the medicine that suits me. The least endorsed items were: I cannot afford either the time or money to get the medicines and I cannot manage so many medicines. Eighteen patients used the free text box, in which concerns about the effects of the medicine or stopping it and the nature of different brands or delivery devices for the same medicine were among the main medicines issues reported.

Results from the correlation analysis showed that MMWFU scores were positively correlated with MMAS4 $(\mathrm{r}=0.59 ; \mathrm{p}<0.001)$ and the BMQ-Concerns $(\mathrm{r}=0.39$,
Table I Patient Responses To Making Medicines Work For You Scale $(N=145)$

\begin{tabular}{|l|l|l|}
\hline Items & $\begin{array}{l}\text { N (Yes } \\
\text { Answers) }\end{array}$ & $\%$ \\
\hline $\begin{array}{l}\text { I. My medicine is difficult to use } \\
\text { 2. I cannot manage so many medicines }\end{array}$ & 15 & 14 \\
3. I am not sure if the medicine is really helping & 40 & 9.7 \\
me & & 27.6 \\
4. I have some worries about using the medicine & 43 & \\
5. I sometimes forget to use the medicine & 70 & 29.7 \\
6. I cannot afford either the time or money to & 7 & 48.3 \\
get the medicines & & 4.8 \\
7. I have found my own way to use the medicine \\
that suits me & 62 & 42.8 \\
8. Other medicines issues & 18 & 12.4 \\
\hline
\end{tabular}

$\mathrm{p}<0.001)$ and had a smaller negative correlation with BMQ-Necessity $(r=-0.24 ; \mathrm{p}<0.05)$.

We tested the sensitivity and specificity of the MMWFU using MMAS4 adherent/non-adherent score as a benchmark. Two cut-off scores of the MMWFU were assessed: (i) $0-1=$ adherent, $2-7=$ non-adherent; (ii) $0=$ adherent and 1-7 = non-adherent. For (i) the sensitivity and specificity were $69.5 \%$ and $68.3 \%$, respectively, and for (ii) $100.0 \%$ and $27.0 \%$, respectively.

\section{Discussion}

This pilot study was designed to assess the usefulness of a new medicines' use screener in clinical practice. All the patients found it quick to complete, and the results indicate that it can identify a range of medicines-related issues. The finding that $88 \%$ of this sample indicated at least one medicines-related issue shows that patients are willing to raise these issues when they are presented in this way, which contrasts with the relatively small numbers, who disclose non-adherence when asked directly. ${ }^{2}$ The pattern of correlations with the BMQ and MMAS4 shows that it can provide an indicator of possible adherence level as well as reflecting negative beliefs about medicines, which are known to be associated with non-adherence. The extent of non-adherence in diabetes is well documented but there are still gaps in our understanding of the reasons for this and in how clinicians can best detect and manage them. $^{7}$

There are a number of limitations of this study. Only patients with type 2 diabetes were included and it will be important to establish how well the screener works in other patient groups and settings. The sensitivity and 
specificity of the MMWFU were tested against a selfreport adherence measure and it would be good to test the reliability of the screener with a biomarker of adherence. Also, it will be important to see whether the responses on the MMWFU are affected if the patient knows that these will be seen by the HCP in an upcoming consultation.

Although the main-proposed use of MMWFU is to drive a discussion with the HCP, these results indicate that it can serve as a fairly sensitive and specific indicator of non-adherence and its determinants. Future studies will be needed to establish (i) how well it performs in other patient groups and clinical settings, and (ii) the extent to which it can activate a helpful adherence discussion with the HCP.

\section{Disclosure}

Professor John Weinman reports personal fees from Atlantis Healthcare, outside the submitted work. Dr Christina Jackson reports personal fees from Takeda, personal fees from Pfizer, personal fees from Amgen, personal fees from Merck, personal fees from IQVIA, outside the submitted work. Ms Anna Hodgkinson occasional does one-off lectures at King's College London for which she is paid a standard fee and is a member of the London Strategic Clinical Network for Diabetes. The authors report no other conflicts of interest in this work.

\section{References}

1. Khan R, Socha-Dietrich K. Investing in medication adherence improves health outcomes and health system efficiency: adherence to medicines for diabetes, hypertension, and hyperlipidaemia. OECD Health Working Papers, No. 105. Paris: OECD Publishing; 2018 doi:10.1787/8178962c-en

2. Engel T, Ungar B, Ben-Haim G, Levhar N, Eliakim R, Ben-Horin S. Re-phrasing the question: a simple tool for evaluation of adherence to therapy in patients with inflammatory bowel disease. United Eur Gastroenterol J. 2017;5(6):880-886. doi:10.1177/2050640616687838

3. Morisky D, Green L, Levine D. Concurrent and predictive validity of a self-reported measure of medication adherence. Med Care. 1986;24 (1):67-74. doi:10.1097/00005650-198601000-00007.

4. Voils C, Maciejewski M, Hoyle R, et al. Initial validation of a self-report measure of the extent and reasons for medication nonadherence. Med Care. 2012;50(12):1013-1019. doi:10.1097/MLR.0b013e318269e121

5. Jackson C, Eliasson L, Barber N, Weinman J. Applying COM-B to medication adherence. Eur Health Psychol. 2014;16:7-17.

6. Horne R, Weinman J, Hankins M. The beliefs about medicines questionnaire: the development and evaluation of a new method for assessing the cognitive representation of medication. Psychol Health. 1999;14,1:1-24. doi:10.1080/08870449908407311

7. Polonsky WH, Henry RR. Poor medication adherence in type 2 diabetes: recognizing the scope of the problem and its key contributors. Patient Prefer Adherence. 2016;10:1299-1307. doi:10.2147/PPA. S106821
Patient Preference and Adherence

\section{Publish your work in this journal}

Patient Preference and Adherence is an international, peer-reviewed, open access journal that focusing on the growing importance of patient preference and adherence throughout the therapeutic continuum. Patient satisfaction, acceptability, quality of life, compliance, persistence and their role in developing new therapeutic modalities and compounds to optimize clinical outcomes for existing disease

\section{Dovepress}

states are major areas of interest for the journal. This journal has been accepted for indexing on PubMed Central. The manuscript management system is completely online and includes a very quick and fair peer-review system, which is all easy to use. Visit http:// www.dovepress.com/testimonials.php to read real quotes from published authors. 